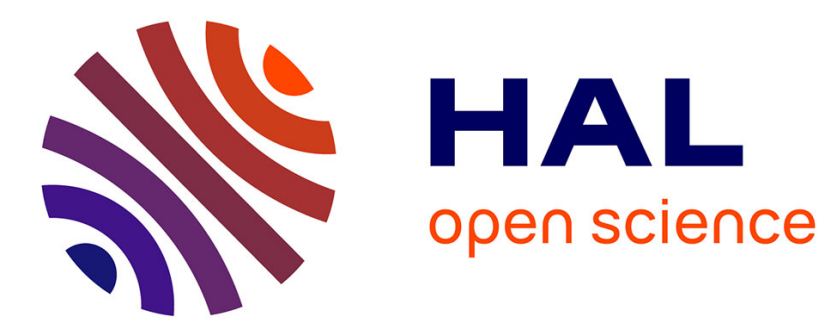

\title{
Suppression of plasma contribution in Femtosecond Degenerate Four-wave Mixing (fs-DFWM) at high intensity
}

\author{
A. Rouzeée, V. Renard, S. Guérin, O. Faucher, B. Lavorel
}

\section{- To cite this version:}

A. Rouzeée, V. Renard, S. Guérin, O. Faucher, B. Lavorel. Suppression of plasma contribution in Femtosecond Degenerate Four-wave Mixing (fs-DFWM) at high intensity. Journal of Raman Spectroscopy, 2007, 38, pp.969. hal-00396997

\section{HAL Id: hal-00396997 https://hal.science/hal-00396997}

Submitted on 19 Jun 2009

HAL is a multi-disciplinary open access archive for the deposit and dissemination of scientific research documents, whether they are published or not. The documents may come from teaching and research institutions in France or abroad, or from public or private research centers.
L'archive ouverte pluridisciplinaire HAL, est destinée au dépôt et à la diffusion de documents scientifiques de niveau recherche, publiés ou non, émanant des établissements d'enseignement et de recherche français ou étrangers, des laboratoires publics ou privés. 


\title{
Suppression of plasma contribution in Femtosecond Degenerate Four-wave Mixing (fs-DFWM) at high intensity
}

\author{
A. Rouzée, V. Renard, S. Guérin, O. Faucher, and B. Lavorel \\ Laboratoire de Physique de l'Université de Bourgogne \\ BP 47870, 21078 Dijon cedex, France
}

\begin{abstract}
Femtosecond Degenerate Four Wave Mixing (fs-DFWM) experiments in $\mathrm{CO}_{2}$ exhibit a strong background due to plasma produced at high intensity $\left(\geq 20 \mathrm{TW} / \mathrm{cm}^{2}\right)$, when significant molecular alignment is likely to arise. This perturbing phenomenon renders the measurements of alignment very difficult. It is shown that the plasma contribution can be avoided by employing perpendicular polarizations for the two pump pulses. The effect is explained on the basis of the different diffraction angles between signals produced by molecular alignment and plasma.
\end{abstract}

Keywords: Femtosecond, degenerate four wave mixing, ionization, molecular alignment

\section{Introduction}

Non-resonant excitation of molecules with high power pulses is a well established technique for aligning anisotropic molecules. ${ }^{1}$ When short laser pulses are used, post-pulse periodic molecular realignment occurs after the pump extinction which is measured either by combining ionization-fragmentation of molecules with an imaging technique ${ }^{1-4}$ or by using nonlinear optical properties of the molecular sample. ${ }^{5,6}$ In the last case, the polarization state and the spatial profile of a probe pulse is perturbed as a consequence of alignment. More precisely, the refractive index shows a time-dependent nonlinear variation due to alignment. ${ }^{5}$, 6 Measuring the perturbation as a function of the delay between the aligning pulse and the probe one enables us to quantify the degree of alignment. Transients grating or degenerate 
four-wave mixing (DFWM) experiments can be also performed to explore molecular alignment. $^{7-9}$ In these experiments, two synchronized crossed pump pulses create a transient grating which diffracts light from a third probe time delayed pulse. The DFWM signal reflects also post-pulse molecular alignment manifested by periodic transients. These transients exhibit a shape corresponding to a rephasing of the rotational wavepacket created by the pump excitation. At low intensity, they are interpreted as resulting from a very tiny periodic realignment of the molecular axis along the pump polarization. By carefully analysing the position and shape of these features, one can obtained accurate information on the molecular structure. ${ }^{10}$ At higher intensity, an efficient molecular alignment is produced. The rotational wavepacket becomes wider leading to a narrowing of the transient shapes and the permanent alignment grows up. Although both effects are responsible for the alteration of the transients, the most apparent feature is the modification of the transient shape due to the interaction with the permanent alignment. ${ }^{5,11}$ In Refs 7-9, the appearance of a strong background was observed with increasing intensity. A part of this background was due to a permanent alignment as mentioned above. At higher intensity, the contribution of a free electron grating has also been suggested in Ref. 8 and invoked in Ref. 9. This free electron grating produces as well a diffraction of the probe pulse which manifests itself as a strong background with a life time of several nanoseconds. As a consequence, the experimental data can not be longer used to measure alignment.

The present work shows that using a polarization grating produced by two crossed perpendicular polarized beams allows us to separate the alignment signal from the plasma one and makes possible quantitative measurement of alignment at high intensity.

\section{Experimental setup}

The experimental setup is based on Femtosecond Degenerate Four-wave Mixing (fs-DFWM) or femtosecond transient grating spectroscopy. ${ }^{12}$ Two synchronized $90 \mathrm{fs}$ pump pulses at 800 $\mathrm{nm}$, derived from a chirp pulse amplified Ti:Sapphire system working at $20 \mathrm{~Hz}$, are focused $(\mathrm{f}=300 \mathrm{~mm})$ and crossed in a molecular sample contained in a gas cell. They produce a transient grating which is monitored by a third time-delayed probe pulse employing the folded boxcars geometry (see insert in Fig. 1). The energy and polarization direction of the two pump pulses are controlled by means of a half wave plate and a polarizer. The amount of diffracted light from the probe pulse is spatially filtered with a pinhole along the phase matching direction and is recorded with a photomultiplier as a function of the time delay. The sample is 
$\mathrm{CO}_{2}$ gas at room temperature. Experiments have been performed with parallel or perpendicular polarizations for the pump pulses.

\section{Results with parallel polarizations}

In this configuration, the resulting polarization is linear and the two pump pulses create an intensity grating, i.e. the total intensity is periodically modulated in the transverse direction with respect to the propagation one (Fig. 1a). This spatial modulation of the total intensity leads to a similar modulation of the degree of alignment quantified by the expectation value $\left(\left\langle\cos ^{2} \theta\right\rangle-1 / 3\right)$, where $\theta$ is the angle between the molecular axis and the polarization direction. Measurement of aligned molecules interacting with a linear polarized laser field and calculation of the degree of alignment has been reviewed in Ref.1 and references therein. At intensity below saturation of alignment, simulations shows that $\left(\left\langle\cos ^{2} \theta\right\rangle-1 / 3\right)$ is roughly proportional to the total pump intensity. ${ }^{9}$ Consequently, the probe beam sees a sinusoidal modulation of the degree of alignment with the same spatial period as the total intensity, and is diffracted.

The signal given by the detector has an intensity :

$$
I_{\text {signal }} \propto\left(\left\langle\cos ^{2} \theta\right\rangle-\frac{1}{3}\right)^{2} \text {. }
$$

To calculate signal (1), the time-dependent Schrödinger equation is solved ${ }^{5}$ to find the value of $\left\langle\cos ^{2} \theta\right\rangle$ at any pump-probe time delay.

The interpretation is based on the nonlinear optical properties of aligned molecules. Indeed, a sample of aligned molecules presents a nonlinear refractive index proportional to the pump intensity (the nonlinear part is proportional to $\left\langle\cos ^{2} \theta\right\rangle-1 / 3$ ). Since the pump intensity is modulated by optical interferences, a spatial modulation of refractive index is formed as shown in Fig. 1a. The resulting phase grating signal is directly related to post-pulse alignment, as predicted by Eq.1. Moreover, due to the spatial beam-profile of the pump pulse, the spatially averaged alignment produced by an on-axis total pump intensity $2 \mathrm{I}_{0}$ is roughly equivalent to that produced by a top-hat distribution with intensity $\mathrm{I}_{0}$.

Fig. 2 shows some recordings with increasing pump intensity. At an intensity of $18 \mathrm{TW} / \mathrm{cm}^{2}$ for each pump (maximum value at the propagation axis), the DFWM signal is very similar to that observed with other experimental techniques. It reflects a small but visible alignment of 
the $\mathrm{CO}_{2}$ axis along the pump polarization through the distorted transient shape. At $36 \mathrm{TW} / \mathrm{cm}^{2}$ a noisy baseline appears, whereas at $70 \mathrm{TW} / \mathrm{cm}^{2}$ the alignment transients disappear. At this intensity, only a fluctuating background signal is observed for a positive delay. This strong structure less and noisy signal comes from the diffraction of the probe beam by the refraction index grating formed by the periodic density of free electrons after ionization of neutral molecules (free electrons cause a decrease of the refractive index, Fig. 1a). This index grating lasts at least a few nanoseconds and produces a constant signal at the time scale investigated in this work. As seen in Fig.2c, alignment is no longer visible in the experimental traces. To overcome this issue, another polarization configuration could be implemented. This is described in the next section.

IV. Results with perpendicular polarizations

For perpendicular pump polarizations, let say one pump at $-45^{\circ}$ and the other at $+45^{\circ}$ with respect to the previous polarization axis (sect. III), the field resulting in the region of spatial overlap is in general elliptically polarized. The polarization of the total electric field depends on the position and is periodic along the transverse direction, resulting in a polarization grating in this direction. Within a fringe, the polarization varies from linear, circular (right), linear (along the perpendicular axis), and circular (left), with intermediate elliptic polarization (Fig. 1b).

It has been shown ${ }^{9}$ that the quantity relevant in the case of perpendicular polarizations is also $\left(\left\langle\cos ^{2} \theta\right\rangle-\frac{1}{3}\right)$.

The average intensity (averaged over an optical cycle) is constant over the interaction volume, except for the overall Gaussian distribution. Nevertheless, the electric field magnitude varies periodically along the transverse coordinate and is maximum for the linear polarizations and minimum for the circular ones. Whereas the ionization process is slightly dependent on the polarization state, it is mainly a function of the instantaneous intensity proportional to the electric field squared. Consequently, the ionization rate of molecules and the free electron density follows the periodic distribution of the electric field amplitude rather than the polarization distribution (Fig. 1b). A phase grating due to the free electrons is formed as in the parallel case. But the periodicity is different than the periodicity of the linear case. As mentioned above, the ionization rate is nearly the same for the two linear and circular polarizations. The phase grating has thus a periodicity twice that of the parallel case. The 
probe pulse is diffracted along a different angle (twice that of the parallel case) and does not reach the detector. The experiment is therefore not sensitive to the electron density grating but only to the alignment grating.

As an example, Fig. 3 shows a typical signal recorded at high intensity. In contrast to Figs. 2 (b-c), the experimental signal is now free from the strong background due to the plasma formation. In the present case, experimental data allows us to study and quantify alignment at high intensity, even in the presence of ionisation. As in the case of the polarization technique, the saturation of the signal due to ionization should be accounted for at very high intensity. ${ }^{13}$ The experimental data have been simulated by using the same routine than for parallel polarizations. Some results are shown in Fig. 4. In the case of perpendicular polarizations, the intensity introduced in the calculation has to be compared to $1 / 2$ times the maximum intensity of each pump beam. ${ }^{9}$ This factor includes the spatial averaging over the beam-profile. The degree of alignment, quantified by $\left\langle\cos ^{2} \theta\right\rangle$, is directly deduced from simulations and is depicted in Fig. 4.

\section{Conclusion}

In conclusion, we have shown that the limitation of DFWM experiments by ionisation at high intensity due to a free electron density grating can be overcome by the means of a configuration with two perpendicular polarized pump pulses. The signal coming from the electron grating is created at an angle twice that the one coming from alignment. This angular separation is used to reject the plasma signal. Experiments can thus be performed at higher intensity to deduce the degree of alignment of molecules such as $\mathrm{CO}_{2}$. It is important to note that using simultaneously the plasma and alignment signals can also lead to interesting applications. For example, it has been recently shown that it is possible to calibrate ionization rate of molecules from their alignment signal. ${ }^{14}$

\section{Acknowledgments}

This work was supported by the Conseil Régional de Bourgogne, the ACI photonique, and the CNRS. 
References

1. $\quad$ Stapelfeldt H ,Seideman T Rev. Mod. Phys. 2003; 75: 543-57.

2. Sakai H, Safvan CP, Larsen JJ, Hilligsoe KM, Hald K ,Stapelfeldt H J. Chem. Phys. 1999; 110: 10235-8.

3. $\quad$ Rosca-Pruna F ,Vrakking MJJ Phys. Rev. Lett. 2001; 87: 153902/1-4.

4. Dooley PW, Litvinyuk IV, Lee KF, Rayner DM, Spanner M, Villeneuve DM ,Corkum PB Phys. Rev. A 2003; 68: 23406-23418.

5. Renard V, Renard M, Guérin S, Pashayan YT, Lavorel B, Faucher O ,Jauslin HR Phys. Rev. Lett. 2003; 90: 153601/1-4.

6. $\quad$ Renard V, Faucher O ,Lavorel B Opt. Lett. 2005; 30: 70-72.

7. $\quad$ Comstock M, Senekerimyan V ,Dantus M J. Phys. Chem. A 2003; 107: 8271-8281.

8. $\quad$ Stavros VG, Harel E, Leone SR J. Chem. Phys. 2005; 122: 64301-1-9.

9. $\quad$ Rouzée A, Renard V, Guérin S, Faucher O ,Lavorel B eprint quant-ph /0609155:

10. Frey HM, Beaud P, Gerber T, Mischler B, Radi PP ,Tzannis AP Applied Physics B Lasers and Optics 1999; B68: 735-9.

11. Lavorel B, Tran H, Hertz E, Faucher O, Joubert P, Motzkus M, Buckup T, Lang T, H. S, Knopp G, Beaud P ,Frey HM C. R. Physique 2004; 5: 215-229.

12. Brown EJ, Qingguo-Zhang, Dantus M J. Chem. Phys. 1999; 110: 5772-88.

13. Rouzee A, Renard V, Lavorel B ,Faucher O Journal of Physics B Atomic, Molecular and Optical Physics 2005; 38: 2329-35.

14. Loriot V, Hertz E, Rouzee A, Sinardet B, Lavorel B ,Faucher O Optics Letters 2006; 31: 2897-9. 


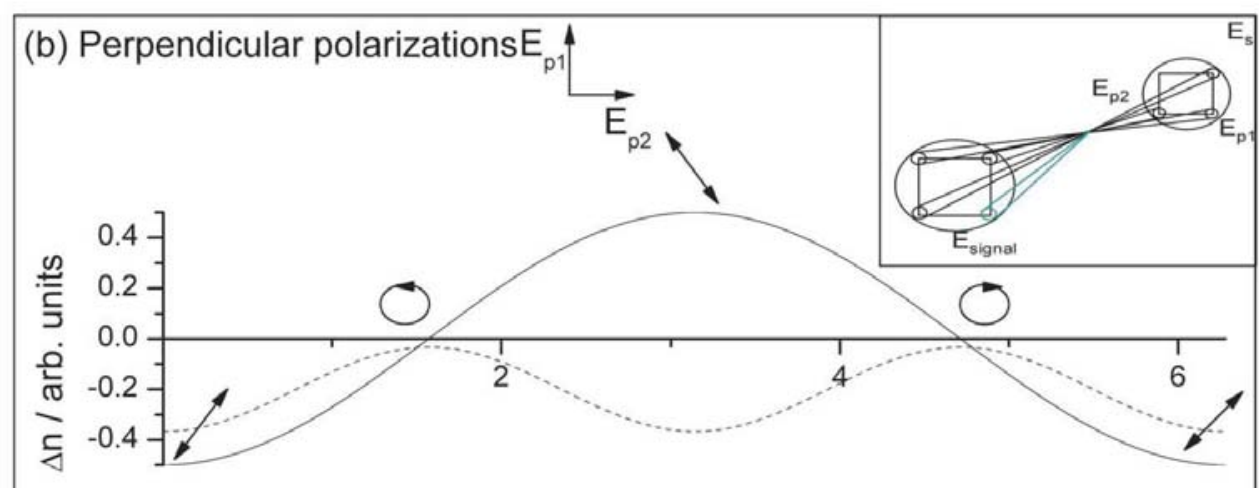

(a) Parallel polarizations $E_{p 1} \uparrow \uparrow E_{p 2}$

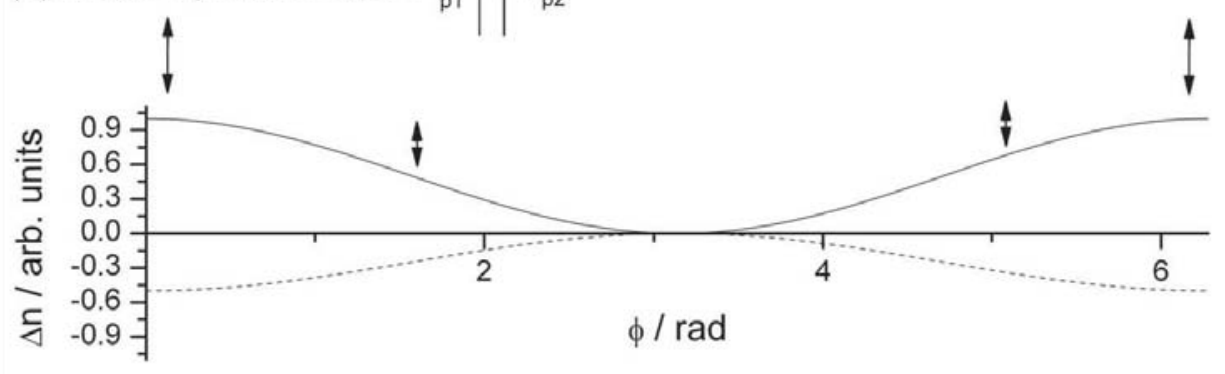

Figure 1: Descriptive of the spatial modulation in the plane wave of the nonlinear refractive index $\Delta \mathrm{n}$ resulting from the aligned molecules (full lines) and the free electrons (dashed lines) versus the relative phase between the two pump fields $E_{P 1}$ and $E_{P 2}$. The arrows and circles correspond to a linear and a circular polarization of the total pump field, respectively. The insert shows the folded boxcars implementation, with $\mathrm{E}_{\mathrm{S}}$ and $\mathrm{E}_{\text {signal }}$ the probe and signal electric fields, respectively. 


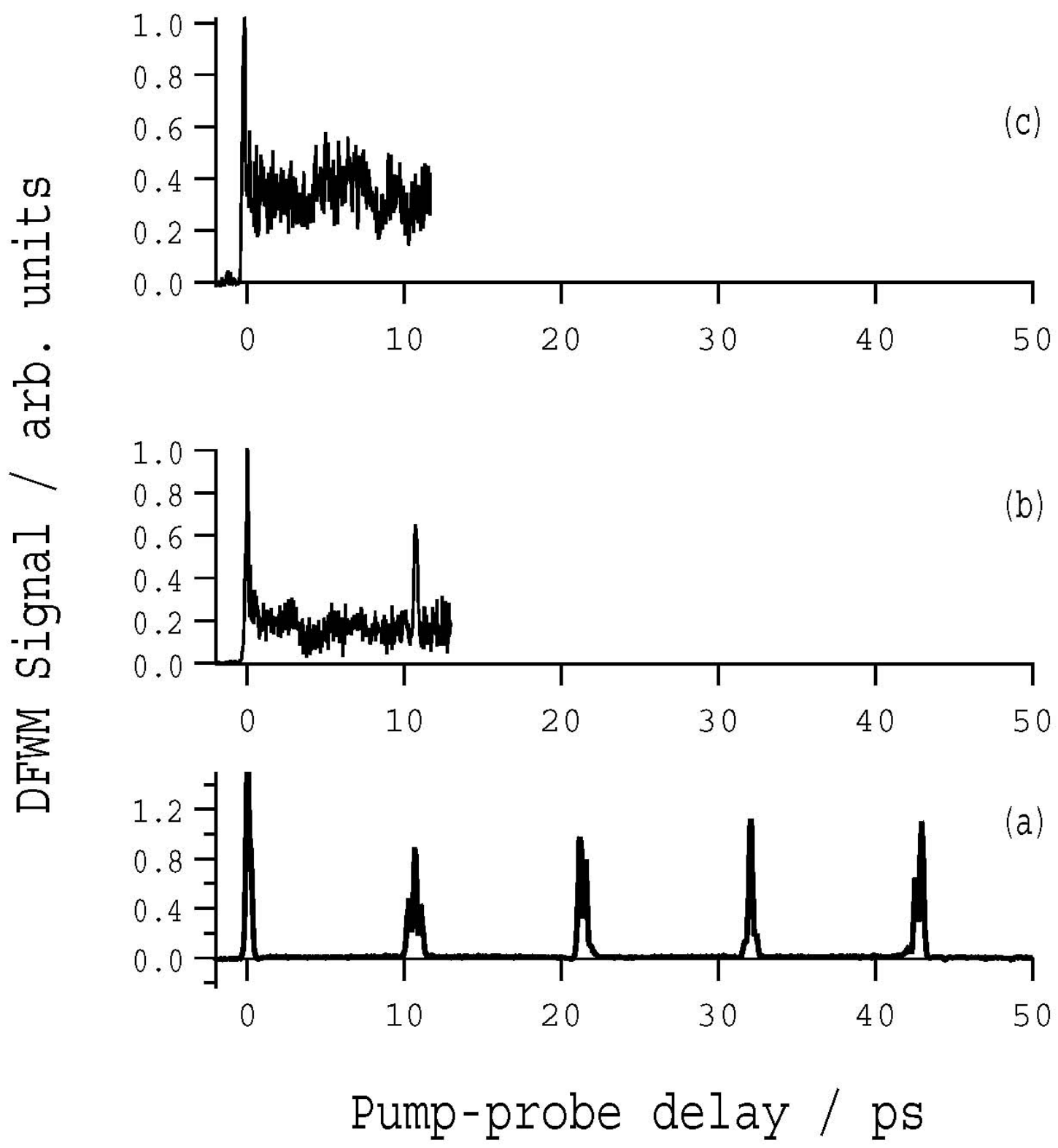

Figure 2: Evolution of the DFWM signal recorded with parallel pump polarizations at room temperature and at 100 mbar pressure. The intensity for each pump is 18 (a), 36 (b), and $70 \mathrm{TW} / \mathrm{cm} 2$ (c). 


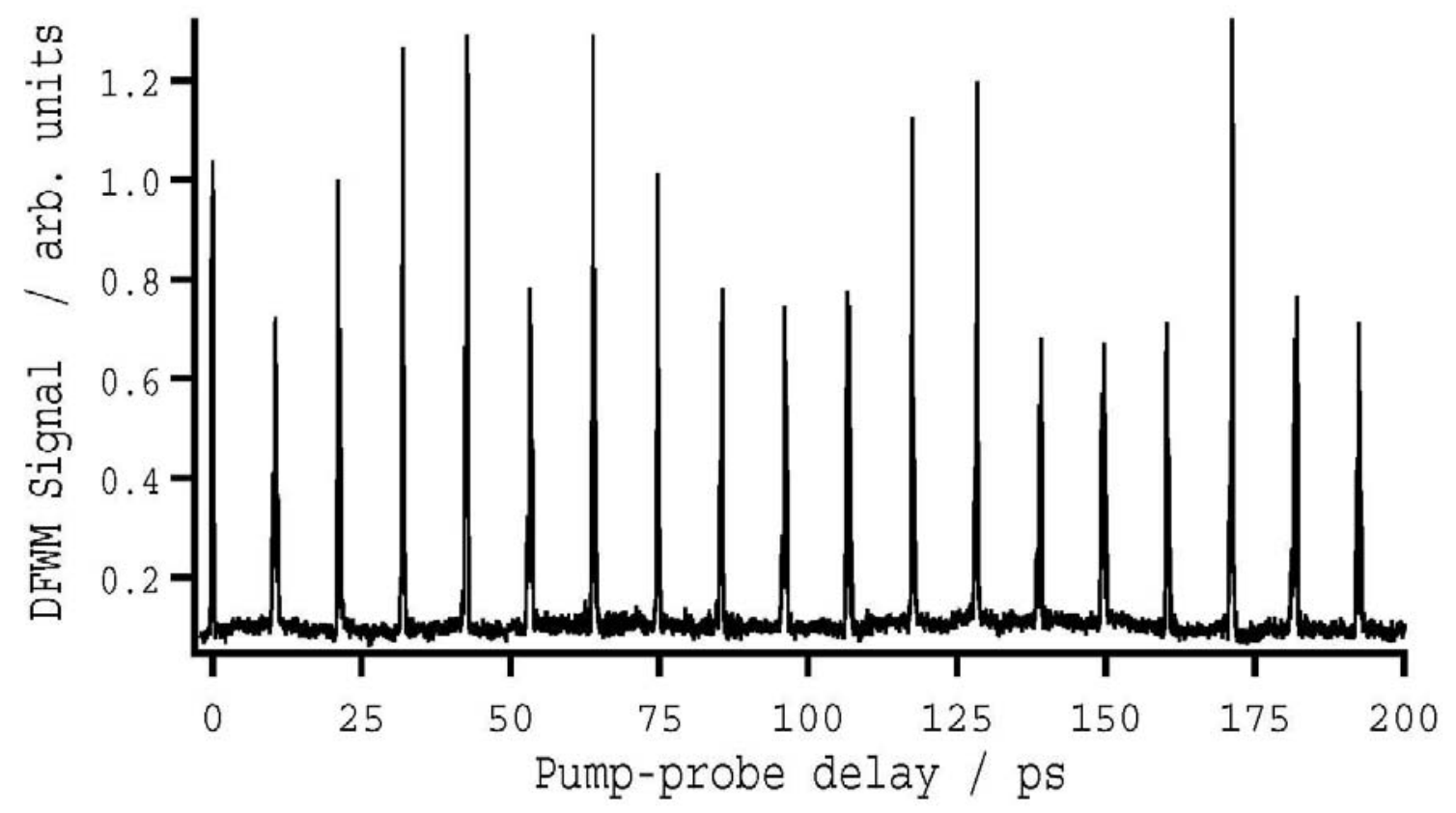

Figure 3: DFWM signal recorded as a function of probe-pump time delay in $\mathrm{CO}_{2}$ at room temperature for perpendicular pump polarizations. Pressure is 6 mbar and measured peak intensity of each pump is $46 \mathrm{TW} / \mathrm{cm}^{2}$. 

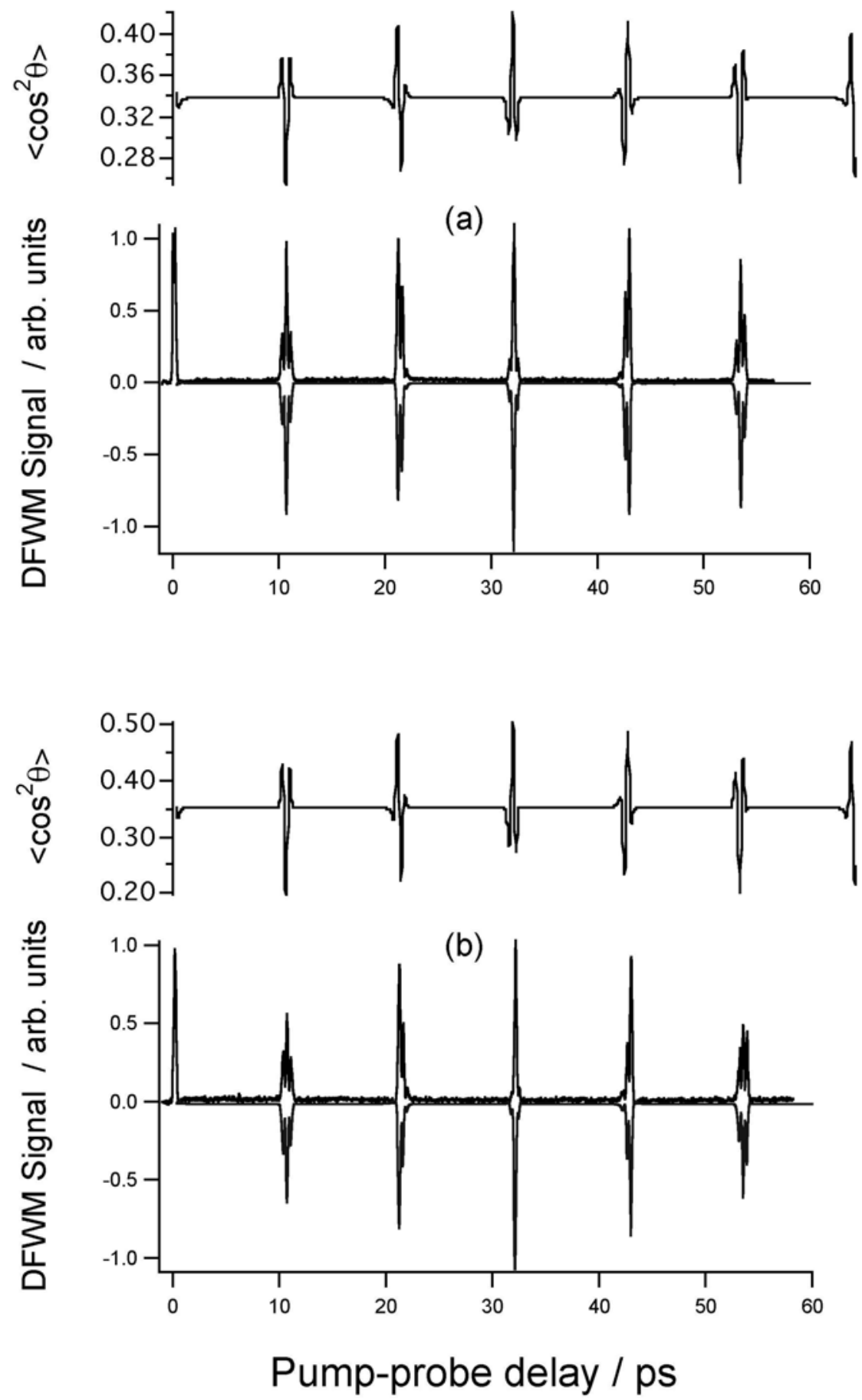

Figure 4: DFWM signal recorded as a function of probe-pump time delay in $\mathrm{CO}_{2}$ at room temperature for perpendicular polarizations. The pressure is $30 \mathrm{mbar}$ and experimental pump peak intensities are respectively $56 \mathrm{TW} / \mathrm{cm}^{2}$ (a) and $100 \mathrm{TW} / \mathrm{cm}^{2}$ (b). The numerical simulations are shown inverted for $20 \mathrm{TW} / \mathrm{cm}^{2}$ (a) and $40 \mathrm{TW} / \mathrm{cm}^{2}$ (b). The value of $\left\langle\cos ^{2} \theta\right\rangle$ is also shown in each case. 